\title{
Using neoadjuvant therapy for breast cancer in clinical practice: when and how?
}

\author{
Debu Tripathy
}

Published online: 22 March 2012

(C) Springer Science+Business Media, LLC. 2012

The roles of neoadjuvant therapy both as a standard therapeutic approach and a research tool are very closely tied and have yielded insights into both breast cancer biology and new drug development $[1,2]$. From a clinician's standpoint, however, the key questions that arise revolve around the clinical advantages of neo-adjuvant therapy in terms of surgical outcomes, long-term recurrence/mortality and decision-making. At the current time, the main advantage of neoadjuvant therapy is to improve the odds of adequate surgical resection, particularly breast-conserving surgery $[3,4]$. Beyond that, long-term outcomes or clinical decision-making do not appear to be affected by the sequence of systemic therapy in relation to definitive surgery. It is becoming clear that breast cancer is heterogeneous. Genomically defined subsets, which map to some extent with hormone and HER2 receptor subtypes, exhibit differential recurrence hazard reductions with systemic therapies-and this concept also applies to neoadjuvant therapy response as well [5]. But there is also clinical diversity, for example, contrasting rapid onset inflammatory cancer to a more indolent cancer that has been clinically present for several years. Therefore, current neoadjuvant treatment paradigms must incorporate the base of evidence from large scale clinical trials as well as the biological context of the disease. The reports contained in this special issue of Breast Cancer Research and Treatment highlight current trends and results that are shaping both patient care and research in this area.

The report by Lin et al.[6] demonstrate biological differences between interval and screen-detected cancers

D. Tripathy $(\bowtie)$

Norris Comprehensive Cancer Center, University of Southern

California, 1441 Eastlake Avenue, \# 3429, Los Angeles,

CA 90033, USA

e-mail: tripathy@usc.edu treated on a prospective trial, even though the number of screening-detected cancers was small, as would be typical in a neoadjuvant trial where the majority of patients were not undergoing screening. As expected, interval cancers had a tendency to be hormone receptor-negative and of higher grade, whereas screen-detected cancers were all of the luminal intrinsic subtypes by gene expression array. Nevertheless, this report points to the need to stratify patients by known characteristics associated with response in clinical trials and to use established factors in routine clinical practice. For example, it is now clear that patients with hormone receptor-positive cancer have less response to neoadjuvant therapy and lower pathologic complete response (pCR) rates. Receptor status might therefore affect the decision regarding the timing of chemotherapy, especially if breast-conserving surgery is driving the decision. Several phenotypes that are also related to hormone receptor content, but appear to have independent prognostic factors for response, such as proliferation index and risk score by gene profile assays are the next markers on the horizon that could enter into this decision-making if tumor size reduction is a main goal of therapy [7, 8].

Is there an optimum neoadjuvant regimen? Should nonstandard adjuvant regimens that yield high pCR rates be adopted into routine practice? Until neoadjuvant trials are powered for long-term outcome, at the current time, it is important to remember that the most important benefit of neoadjuvant therapy is its "adjuvant" effect on long-term recurrence and mortality. Therefore, an evidence-based approach would dictate the use of proven standard adjuvant regimens in the neoadjuvant setting. However, trials that bridge pCR rates in relevant subsets to long-term outcome could serve as the basis for approvals of new drugs and regimens and eventually shorten regulatory approval times [9]. Some long-standing standards may need to be 
questioned based in findings in neoadjuvant trials-for example, the use of sequential adjuvant hormonal therapy after chemotherapy as opposed to concurrent use has now become the standard based on trials using tamoxifen even these have not been definitive $[10,11]$. However, concurrent use of aromatase inhibitors, which may interact with chemotherapy in a manner distinct from that of tamoxifen, has never been formally tested. One of the articles in this supplement provides support for such studies, in showing a significant increase in pCR rate with concurrent letrozole compared to chemotherapy alone, and this may raise the larger questions of concurrent hormonal and chemotherapy [12]. Newer paradigms of dual blockade of the HER2 pathway are uniformly showing benefits without much added toxicity of trastuzumab combined with pertuzumab or lapatinib although for now, these will need to be verified in prospective trial as they are testing novel agents [13, 14]. However, other questions specific the neoadjuvant setting such as the value of anthracycline versus non-anthracyline in combination trastuzumab in achieving a higher degree of response has been suggested, but may need formal testing specifically in the neoadjuvant setting where response is linked to long-term outcome [15].

This issue also contains reports of novel predictive markers such as p53 mutation status, the extent tumor infiltrating lymphocyte and the cell death pathway mediator Serpin3 [16-18]. However, markers predictive of specific agents and regimens are needed for clinical decisionmaking and will require further evaluation in both neoadjuvant and adjuvant trials. This is critical since early predictive biomarkers that have arisen from the laboratory are not always being confirmed in larger scale clinical trials, for example, the truncated HER2 receptor p95 that is associated with resistance to trastuzumab in laboratory models and small clinical series are not holding up when analyzed in the context of larger randomized neoadjuvant trials $[19,20]$. However, clinical decision-making will require treatment-specific predictors that are validated and consistent across studies, with the recognition that some predictors or classifiers may apply to response and not metastasis and survival, and as such will need to be applied in the proper clinical context. If clinical response is the key driver for making a decision in a specific case, then the indices used for this outcome should be employed.

Among the greatest potential advantage of neoadjuvant therapy would be the use of degree of response to adjust therapy to improve long-term outcome. In the GeparTrio study, the strategy of changing chemotherapy following clinical response assessment at two cycles did not improve pCR rate, but interestingly, on longer term follow-up, there is now a disease-free survival based on response-guided therapy in non-responders [21]. These are the types of trials that hold the potential to improve outcome through the information gained during the course of therapy. Ultimately, this approach can lead to new discovery of agents and regimens and the appropriate classifiers to optimize candidates who will be benefit the most.

\section{References}

1. Kaufmann M, Hortobagyi G, Goldhirsch A et al (2006) International expert panel on the use of primary (preoperative) systemic treatment of operable breast cancer: review and recommendations. Recommendations from an international expert panel on the use of neoadjuvant (primary) systemic treatment of operable breast cancer: an update. J Clin Oncol 24:1940-1949

2. Wolff AC, Berry D, Carey L et al (2008) Research issues affecting preoperative systemic therapy for operable breast cancer. J Clin Oncol 26:806-813

3. Bear HD, Anderson S, Smith RE et al (2006) Sequential preoperative or postoperative docetaxel added to preoperative doxorubicin plus cyclophosphamide for operable breast cancer: National Surgical Adjuvant Breast and Bowel Project Protocol B-27. J Clin Oncol 24:2019-2027

4. Liu SV, Melstrom L, Yao K et al (2010) Neoadjuvant therapy for breast cancer. J Surg Oncol 101:283-291

5. Hatzis C, Pusztai L, Valero V et al (2011) A genomic predictor of response and survival following taxane-anthracycline chemotherapy for invasive breast cancer. JAMA 305:1873-1881

6. Lin C, Buxton MB, Moore D et al (2012) Locally advanced breast cancers are more likely to present as Interval Cancers: results from the I-SPY 1 TRIAL (CALGB 150007/150012, ACRIN 6657, InterSPORE Trial). Breast Cancer Res Treat. doi: 10.1007/s10549-011-1670-4

7. Zambetti M, Mansutti M, Gomez P et al (2012) Pathological complete response rates following different neoadjuvant chemotherapy regimens for operable breast cancer according to ER status, in two parallel, randomized phase II trials with an adaptive study design (ECTO II). Breast Cancer Res Treat. doi:10.1007/ s10549-011-1660-6

8. Esserman LJ, Berry DA, Cheang MC et al (2011) Chemotherapy response and recurrence-free survival in neoadjuvant breast cancer depends on biomarker profiles: results from the I-SPY 1 TRIAL (CALGB 150007/150012; ACRIN 6657). Breast Cancer Res Treat. doi:10.1007/s10549-011-1895-2

9. Esserman LJ, Woodcock J (2011) Accelerating identification and regulatory approval of investigational cancer drugs. JAMA 306: 2608-2609

10. Albain KS, Barlow WE, Ravdin PM et al (2009) Adjuvant chemotherapy and timing of tamoxifen in postmenopausal patients with endocrine-responsive, node-positive breast cancer: a phase 3, open-label, randomised controlled trial. Lancet 374:2055-2063

11. Bedognetti D, Sertoli MR, Pronzato P et al (2011) Concurrent vs sequential adjuvant chemotherapy and hormone therapy in breast cancer: a multicenter randomized phase III trial. J Natl Cancer Inst 103:1529-1539

12. Mohammadianpanah M, Ashouri Y, Hoseini S et al (2012) The efficacy and safety of neoadjuvant chemotherapy \pm letrozole in postmenopausal women with locally advanced breast cancer: a randomized phase III clinical trial. Breast Cancer Res Treat

13. Baselga J, Bradbury I, Eidtmann H et al (2012) Lapatinib with trastuzumab for HER2-positive early breast cancer (NeoALTTO): a randomised, open-label, multicentre, phase 3 trial. Lancet 379:633-640

14. Gianni L, Pienkowski T, Im YH et al (2012) Efficacy and safety of neoadjuvant pertuzumab and trastuzumab in women with 
locally advanced, inflammatory, or early HER2-positive breast cancer (NeoSphere): a randomised multicentre, open-label, phase 2 trial. Lancet Oncol 13:25-32

15. Bayraktar S, Gonzalez-Angulo AM, Lei X et al (2011) Efficacy of neoadjuvant therapy with trastuzumab concurrent with anthracycline-and nonanthracycline-based regimens for HER2positive breast cancer. Cancer. doi:10.1002/cncr.26555

16. Glück S, Ross JS, Royce M et al (2012) TP53 genomics predict higher clinical and pathologic tumor response in operable earlystage breast cancer treated with docetaxel-capecitabine-trastuzumab. Breast Cancer Res Treat. doi:10.1007/s10549-011-1412-7

17. Ono M, Tsuda H, Shimizu C et al (2012) Tumor-infiltrating lymphocytes are correlated with response to neoadjuvant chemotherapy in triple-negative breast cancer. Breast Cancer Res Treat. doi:10.1007/s10549-011-1554-7

18. Collie-Duguid ESR, Sweeney K, Stewart KN et al (2012) SerpinB3, a new prognostic tool in breast cancer patients treated with neoadjuvant chemotherapy. Breast Cancer Res Treat. doi: 10.1007/s10549-011-1625-9

19. Scaltriti M, Rojo F, Ocana A et al (2007) Expression of p95HER2, a Truncated Form of the HER2 receptor, and response to anti-HER2 therapies in breast cancer. J Natl Cancer Inst 99:628-638

20. Loibl S, Bruey J, von Minckwitz G et al (2011) Validation of p95 as a predictive marker for trastuzumab-based therapy in primary HER2-positive breast cancer: a translational investigation from the neoadjuvant GeparQuattro study. J Clin Oncol 29(suppl):abstr 530

21. Von Minckwitz G, Blohmer JU, Costa S et al (2011) Neoadjuvant chemotherapy adapted by interim response improves overall survival or primary breast cancer patients-results of the GeparTrio trial. Program and abstracts of the 34th Annual San Antonio Breast Cancer Symposium, December 6-10, San Antonio, Texas, Abstract S3-2 